\title{
Location of glycosidases and two xylanases in strictly anaerobic rumen fungi
}

\author{
B Gaillard-Martinie 1, A Breton 1, B Kherratia 1, M Dusser 2 \\ 1 INRA, Laboratoire de Microbiologie, C.R. de Clermont-Ferrand-Theix 63122 Saint Genès-Champanelle \\ 2 Faculté de Pharmacie, Laboratoire de Botanique, 63000 Clermont-Ferrand, France
}

Glycosidase and xylanase activities have been located with fluorescent conjugates and indirect immunofluorescence in the thallus of two species of strictly anaerobic ruminal fungi : Neocallimastix frontalis and Caecomyces communis. For Neocallimastix frontalis, xylanase I has been localized by transmission electron microscopy after immunogold. $\beta$-Dglucosidase, $\beta$-D-fucosidase, $\beta$-D-xylosidase, $\beta$-D-cellobiopyranosidase, $\alpha$-L-arabinofuranosidase have been detected with 4-methyllumbelliferyl-glycosides, fluorescent conjugates which give a blue fluorescence under UV at $365 \mathrm{~nm}$ after enzymatic hydrolysis. Enzyme activities occurred from the initiation of spore germination and were present in cell-walls, cytoplasm of vegetative cells and in the rhizoids or the vesicles. They disappeared during sporulation and were absent in zoospores. $\beta$-D-galactosidase and $\alpha-L-$ arabinopyranosidase were inexistant.

The localization of xylanases I and III on the whole thalli and on semi-fine sections (2 $\mu \mathrm{m})$ were observed under indirect immunofluorescence using $N$. frontalis antixylanase rabbit antibodies. The best visualization of the antigene-antibody complex was obtained with goat secondary antibodies or A protein conjugated with FITC. Xylanases I and III were apparent on the surface of the thalli of the two fungal species studied and disappeared in sporulating thalli.

In electron microscopy, on ultrafine sections $(70 \mathrm{~nm})$ of Neocallimastix frontalis ; xylanase I has been localized in immunogold by visualization of the antigene-antibody complex by goat secondary antibodies conjugated with colloïdal gold. Xylanase I was strongly associated with sporangia cell-walls and was localized in the cytoplasm of rhizoids.

Glycosidase and xylanase activities occurred throughtout the whole thallus of both $N$. frontalis and C. communis as from zoospore germination, disappeared before sporulation and were absent in zoospores. Similarity results obtained with 3 techniques used and the cross reactions between anti-xylanase antibodies demonstrate the existence of analogies in structure among enzymes of the anaerobic fungal species and confirm the functional homogeneity of this group. 